\title{
A computationally efficient detector for MIMO systems
}

\author{
Samer Alabed \\ College of Engineering and Technology, American University of the Middle East, Kuwait
}

Article Info

Article history:

Received Sep 13, 2018

Revised Apr 9, 2019

Accepted Apr 28, 2019

Keywords:

MIMO systems

MIMO detectors

Zero forcing decoder

Maximum likelihood decoder

Sphere decoder

Minimum mean squared error

\begin{abstract}
AIn this work, a newly designed multiple-input multiple-output (MIMO) detector for implementation on software-defined-radio platforms is proposed and its performance and complexity are studied. In particular, we are interested in proposing and evaluating a MIMO detector that provides the optimal trade-off between the decoding complexity and bit error rate (BER) performance as compared to the state of the art detectors. The proposed MIMO decoding technique appears to find the optimal compromise between competing interests encountered in the implementation of advanced MIMO detectors in practical hardware systems where it i) exhibits deterministic decoding complexity, i.e., deterministic latency, ii) enjoys a good complexity-performance trade-off, i.e., it keeps the complexity considerably lower than that of the maximum likelihood detectors with almost optimal performance, iii) allows fully parameterizable performance to complexity trade-off where the performance (or complexity) of the MIMO detector can be adaptively adjusted without the requirement of changing the implementation, iv) enjoys simple implementation and fully supports parallel processing, and v) allows simple and efficient extension to soft-bit output generation for support of turbo decoding. From the simulation results, the proposed MIMO decoding technique shows a substantially improved complexity-performance trade-off as compared to the state of the art techniques.
\end{abstract}

Copyright (c) 2019 Insitute of Advanced Engineeering and Science. All rights reserved.

\section{Corresponding Author:}

Samer Alabed,

Assistant professor,

Department of Electrical Engineering, College of Engineering and Technology, American University of the Middle East, Kuwait.

Phone: +965 22251400 Ext.: 1790

Email: Samer.Al-Abed@aum.edu.kw

\section{INTRODUCTION}

In the last decade, cooperative and multiple-input multiple-output (MIMO) techniques have been extensively studied as their improvements in performance do not require additional power or frequency spectrum [1-13]. In this work, the performance of existing linear and nonlinear decoders [2, 14-20] for MIMO systems is compared with the newly proposed decoder that is particularly suitable for implementation on software-defined-radio architectures. The maximum likelihood (ML) decoder is the optimal detector for MIMO systems $[2,15]$. In this decoder, a search over all possible combination of transmitted symbol vectors is performed. The ML detection proves to be optimal, however, at the cost of high complexity which increases exponentially with the increase of the modulation size and the number of transmit antennas $[15,16]$. On the other hand, linear detectors such as the zero forcing (ZF) and minimum mean squared error (MMSE) detectors are the simplest and widely used detectors with reasonably lower bit error rate (BER) performances at very low computational complexity $[2,4,17,18]$. Correspondingly, the vertical Bell laboratories layered space-time (V-BLAST) technique uses an iterative detector that implements the concept of successive interference cancellation (SIC) to find a good trade-off between complexity and performance [2,18-20]. SIC decoder can be 
further improved by incorporating appropriate ordering of the symbols, i.e., first decoding the symbols that exhibit small estimation error before detecting the weaker symbols.

In this work, we are interested in implementing, developing and evaluating a MIMO detector that provides the optimal trade-off between the decoding complexity and BER performance as compared to the state of the art detectors. Therefore, we introduce a new MIMO decoding technique which i) enjoys a good complexity-performance trade-off, ii) allows fully parameterizable performance configuration, in the sense that, the performance of the MIMO detector can be adaptively adjusted without the requirement of changing the implementation, iii) enjoys simple implementation and fully supports massive parallel processing, iv) exhibits a fixed complexity, i.e., unlike the popular sphere decoder, the decoding complexity is deterministic and does not depend on the particular realizations of fading or noise environments, and v) allows natural extension to soft-bit decoding required for modern channel decoders.

\section{SYSTEM MODEL}

Let us consider a MIMO system with $n T x$ transmit and $n R x$ receive antennas as illustrated in Figure 1 and assume frequency non-selective flat fading channels. If a signal vector $\mathbf{x}$ is sent from the transmit antenna array where symbol $x_{j}$ emitted from the $j$ th transmit antenna and $y_{i}$ is received by the $i$ th antenna, then the signal at the receive antennas can be expressed as

$$
\mathbf{y}=\mathbf{H} \mathbf{x}+\mathbf{n}
$$

where $\mathbf{y}=\left[y_{1}, y_{2}, \cdots, y_{n R x}\right]^{T}, \mathbf{x}=\left[x_{1}, x_{2}, \cdots, x_{n T x}\right]^{T}, \mathbf{n}=\left[n_{1}, n_{2}, \cdots, n_{n R x}\right]^{T}$, and $\mathbf{H}$ denotes the MIMO channel matrix which describes the input-output relation. In this representation, $\mathbf{n}$ denotes the noise vector which is modeled as independent, zero-mean, complex Gaussian random variables with unit variance.

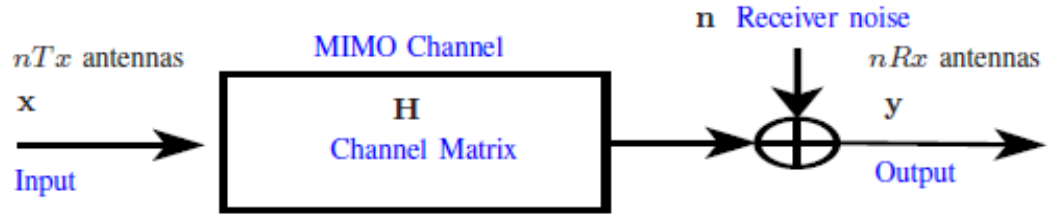

Figure 1. System model of MIMO channel

Let $\mathbf{H}$ be a $n R x \times n T x$ matrix $(n R x \geq n T x)$ with $\operatorname{rank}(\mathbf{H})=n T x$. $\mathbf{H}$ can be decomposed into the QR decomposition, such that

$$
\mathbf{H}=\mathbf{Q R}
$$

where $\mathbf{Q}$ is an $n R x \times n R x$ unitary matrix, $\mathbf{R}$ is an $(n R x \times n T x)$ upper triangular matrix, and $\mathbf{I}_{n T x}$ is an $(n T x \times n T x)$ identity matrix. Making use of the $\mathrm{QR}$ decomposition, we can transform the channel model (1) into an equivalent triangular channel, such that

$$
\tilde{\mathbf{y}}=\mathbf{Q}^{H} \mathbf{y}=\mathbf{Q}^{H}(\mathbf{H} \mathbf{x}+\mathbf{n})=\mathbf{R} \mathbf{x}+\tilde{\mathbf{n}}
$$

where $\tilde{\mathbf{n}}=\mathbf{Q}^{H} \mathbf{n}$. After preprocessing the received data, model (3) becomes in a triangularized form.

\section{THE PROPOSED RANDOMIZATION BASED MMSE DECODER} following steps:

In this section, let us introduce a new MIMO decoding algorithm where this algorithm carries out the

\subsection{Step 1: Preprocessing (Nulling/Channel equalization)}

Nulling, i.e. channel equalization, is used to remove the channel effect from the received signal vector. This process is performed using MMSE channel matrix inversion. The linear receiver $\mathbf{W}_{\text {MMSE }}$ is computed to minimize the mean squared error (MSE) [2] of the received signal, given by

$$
f_{\mathrm{MMSE}}(\mathbf{x})=\mathrm{E}\left\{\left(\mathbf{W}_{\mathrm{MMSE}} \mathbf{y}-\mathbf{x}\right)\left(\mathbf{W}_{\mathrm{MMSE}} \mathbf{y}-\mathbf{x}\right)^{H}\right\}
$$


where $\mathrm{E}\{\cdot\}$ denotes statistical expectation. Therefore, the equalization matrix $\mathbf{W}_{\mathrm{MMSE}}$ corresponding to the MMSE decoder is expressed as

$$
\mathbf{W}_{\text {MMSE }}=\left(\mathbf{H}^{H} \mathbf{H}+\sigma^{2} \mathbf{I}\right)^{-1} \mathbf{H}^{H}
$$

where $\mathbf{I}$ denotes the identity matrix. According to the principle of linear receivers, $\mathbf{W}_{\mathrm{MMSE}}$ given in (5) is multiplied by $\mathbf{y}$ given in (1) to reconstruct the symbol vector $\mathbf{x}$ by removing the channel effect and suppressing noise enhancement, such that $\hat{\mathbf{x}}_{\mathrm{MMSE}}=\mathbf{W}_{\mathrm{MMSE}} \mathbf{y}$. For convenience of representation and for our derivations in the following, the MMSE decoder can be formulated in another form. Making use of $\mathrm{QR}$ decomposition explained in Sec. 2., $\overline{\mathbf{H}}$ can be expressed as

$$
\overline{\mathbf{H}}=\left[\begin{array}{c}
\mathbf{H} \\
\sigma \mathbf{I}
\end{array}\right]=\left[\begin{array}{l}
\mathbf{Q}_{1} \\
\mathbf{Q}_{2}
\end{array}\right] \overline{\mathbf{R}}=\overline{\mathbf{Q}} \overline{\mathbf{R}}
$$

where the partitioning is such that $\overline{\mathbf{Q}}=\mathbb{C}^{2 n R x \times n T x}, \mathbf{Q}_{1}=\mathbb{C}_{1}^{n R x \times n T x}, \mathbf{Q}_{2}=\mathbb{C}_{2}^{n R x \times n T x}$ and $\overline{\mathbf{R}}=$ $\mathbb{C}^{n T x \times n T x}$. Considering (6), the equalization matrix $\mathbf{W}_{\text {MMSE }}$ corresponding to $\mathbf{y}$ in (1) can be expressed as

$$
\begin{aligned}
\mathbf{W}_{\text {MMSE }} & =\left(\overline{\mathbf{H}}^{H} \overline{\mathbf{H}}\right)^{-1} \mathbf{H}^{H}=\left(\mathbf{H}^{H} \mathbf{H}+\sigma^{2} \mathbf{I}\right)^{-1} \mathbf{H}^{H} \\
& =\left(\overline{\mathbf{R}}^{H} \overline{\mathbf{R}}\right)^{-1} \overline{\mathbf{R}}^{H} \mathbf{Q}_{1}^{H}=\overline{\mathbf{R}}^{-1} \mathbf{Q}_{1}^{H}
\end{aligned}
$$

where $\overline{\mathbf{H}}$ is given in (6). Making use of the equalization matrix (7), the soft-decoded symbol after MMSE decoding becomes

$$
\hat{\mathbf{x}}_{\text {MMSE }}=\mathbf{W}_{\text {MMSE }} \mathbf{y}=\mathbf{x}+\overline{\mathbf{R}}^{-1} \mathbf{Q}_{1}^{H} \mathbf{n}=\mathbf{x}+\mathbf{e}
$$

where $\mathbf{x}$ denotes the true transmitted symbol vector and the estimation error vector $\mathbf{e}=\hat{\mathbf{x}}-\mathbf{x}$ is Gaussian distributed with zero mean and error covariance matrix given by

$$
\mathrm{E}\left\{\mathbf{e e}^{H}\right\}=\sigma^{2}\left(\mathbf{H}^{H} \mathbf{H}+\sigma^{2} \mathbf{I}\right)^{-1}=\sigma^{2}\left(\overline{\mathbf{R}}^{H} \overline{\mathbf{R}}\right)^{-1}
$$

This step is carried out only once and before the randomization is started.

\subsection{Step 2: Generating random vector}

In this step, the decoder generates a number of instances of a random vector $\mathbf{e}_{k},\left\{k=1, \ldots, N o \_\right.$rand $\}$ with mean and variance equal to those of the estimation error e in (9), i.e., $\mathbf{e}_{k} \in \mathcal{N}\left(\mathbf{0}, \sigma^{\mathbf{2}}\left(\overline{\mathbf{R}}^{\mathbf{H}} \overline{\mathbf{R}}\right)^{-\mathbf{1}}\right)$ where No_rand is the number of generated random vectors and $\mathbf{e}_{k}$ denotes the $k$ th generated random vector. From $\left\{\mathbf{e}_{k}\right\}_{k=1}^{N o-r a n d}$, a corresponding set for random vectors is computed according to

$$
\hat{\mathbf{x}}_{k}=\hat{\mathbf{x}}_{\mathrm{MMSE}}+\mathbf{e}_{k} \quad \text { for } \mathrm{k}=1, \ldots, \text { No_rand }
$$

Note that generating more instances of a random vector $e_{k}$ will increase the probability to have one of them as close as possible to the optimal one. By doing this, the overall BER performance will improve.

\subsection{Step 3: Hard decoding}

In this step, the decoder converts for $k=1, \ldots$, No_rand the soft decoded randomized symbol vector $\hat{\mathbf{x}}_{k}$ generating according to (10) to hard decoded symbol vector $\tilde{\hat{\mathbf{x}}}_{k}$ by finding the nearest constellation point for each soft decoded symbol as shown in Figure 2. Note that this is a symbol by symbol processing step performed using the round operation with almost no additional computational complexity.

\subsection{Step 4: Selection}

In this step, the decoder selects among the hard decoded symbol vector $\tilde{\hat{\mathbf{x}}}_{k}$ for $k=1, \ldots, N o \_$rand the vector $\mathrm{x}^{\text {prop. }}$ that maximizes the ML metric, such as

$$
\mathbf{x}^{\text {prop. }}=\arg \min _{\tilde{\mathbf{x}}_{1}, \ldots, \tilde{\hat{\mathbf{x}}}_{\text {No_rand }}}\|\tilde{\mathbf{y}}-\overline{\mathbf{R}} \mathbf{x}\|^{2} .
$$

The above described main procedure in Steps 2-3 can be efficiently implemented using either an iterative or a parallelized implementation as shown in Figure 2. The estimate of a symbol obtained by using MMSE 
filter has a bias or mean and variance. The randomization algorithm says that there is a high probability to get closer to the actual symbol by searching randomly for a symbol set having same mean as our estimated symbol set and within the limits of the variance circle. By doing this, a better estimate of symbols can be found. It is clear that we can improve the performance of the decoder by increasing the number of randomization instances, i.e., the value of No_rand. This, however, comes at the expense of increased decoding complexity. Therefore, the performance (or complexity) of this decoder can be adaptively adjusted by changing the number of randomization instances without the requirement of changing the structure of the implementation, i.e., the performance to complexity trade-off can be adjusted using system parameter No_rand. Furthermore, the proposed algorithm enjoys simple implementation based on the widely used MMSE technique. The proposed algorithm offers a fixed decoding complexity that does not depend on the quality of the received signal vector $\mathbf{y}$.

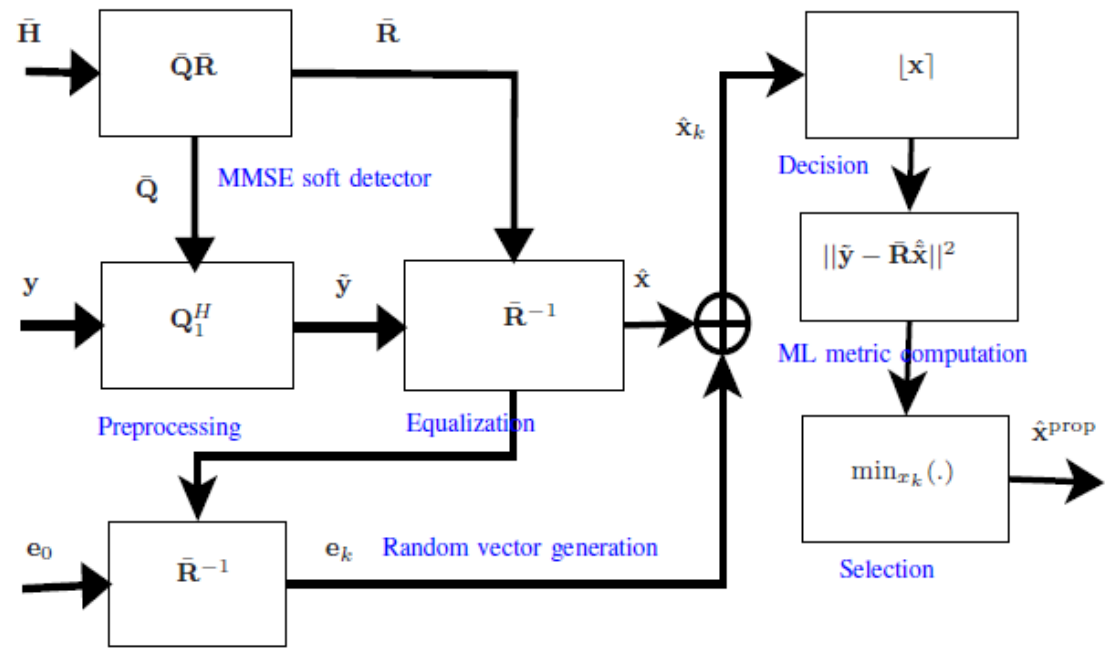

Figure 2. Block diagram of the proposed decoder

\section{SIMULATION RESULTS}

In the simulations, let us considered MIMO systems with independent flat Rayleigh fading channels and either four transmit and four receive antennas, eight transmit and eight receive antennas, or 20 transmit and 20 receive antennas. All MIMO detectors using 4-QAM, 16-QAM, and 64-QAM constellations are compared. In the proposed decoder, the symbols can also be drawn from any M-PSK constellation. In all illustrated figures, it can be observed that the ZF decoder exhibits the worst performance, however, with linear decoding complexity. On the other hand, the curves which enjoy the optimal decoding performance in any of the figures represent the sphere decoder or ML decoder, however, ML decoder suffers from extremely high (exponential) decoding complexity. Any other decoder has a performance and decoding complexity between that of the ML and the ZF decoder. In the following figures, ZF, MMSE, SIC, SD, ML, K, and Rand $=L$ denote the ZF decoder, MMSE decoder, SIC decoder, sphere decoder, ML decoder, K-best decoder with $K^{n T x}$ iterations and the proposed decoder using randomization technique with $L$ iterations. From the figures, it is observed that i) the decoders using MMSE outperform those using ZF due to their robustness with respect to noise enhancement as compared to the ZF decoders, ii) the ML decoder enjoys optimal performance at the cost of very high decoding complexity, iii) the proposed decoder can improve the complexity-performance trade-off where it keeps the complexity considerably lower than that of the ML detectors with almost optimal performance, and iv) the sphere decoder which enjoys the optimal performance does not have a fixed complexity and in specific cases the complexity may be as large as the complexity of the ML decoder, however, some sub-optimal sphere decoders enjoys fixed decoding complexity, e.g., K-best decoder with suboptimal performance as shown in Figure 3 [20]. 


\section{COMPLEXITY-PERFORMANCE TRADE-OFF}

From Figures 3, 4, 5 and 6, both, sphere decoder and ML decoder are optimal and have exactly the same BER performance and the proposed decoder using only 10 iterations outperforms the suboptimal decoders, i.e., ZF, MMSE, and SIC with and without ordering using ZF or MMSE. We emphasize that, in the proposed randomization based decoder, the nulling step, i.e., the matrix inversion step using QRdecomposition, is carried out only once and before the randomization is started as discussed in Sec. 3., while SIC decoder performs the same step every layer [2]. In all investigated decoders, i.e., ZF decoder, MMSE decoder, ML decoder, sphere decoder, SIC decoder, K-best decoder and the proposed randomization based decoder, the QR decomposition stage described in Sec. 2. requires $\mathbf{O}\left((n T x-i)^{3}\right)$ operations. Once the $\mathrm{QR}$ decomposition of $\mathbf{H}$ is obtained, the remainder stages in the SIC decoder at the $i$ th layer, the ZF/MMSE decoder, the ML decoder, K-best decoder, and the proposed decoder require $\mathbf{O}\left((n T x-i)^{2}\right)$ operations, $\mathbf{O}\left((n T x)^{2}\right)$ operations, $\mathbf{O}\left((n T x)^{2.38}\right) M^{n T x}$ operations, $\mathbf{O}\left((n T x)^{2.38}\right) K^{n T x}+n T x \times \mathbf{O}\left((n T x)^{2}\right)$ operations, and $\mathbf{O}\left((n T x)^{2.38}\right)$ No_rand $+\mathbf{O}\left((n T x)^{3}\right)$ operations, respectively, as shown in Table 1. Note that, in the proposed decoder, computing the inverse of the channel matrix, i.e., $\mathbf{H}^{-1}=\mathbf{R}^{-1} \mathbf{Q}^{H}$, requires $\mathbf{O}\left((n T x)^{3}\right)$ after obtaining the QR decomposition of $\mathbf{H}$.

From Figure 3, it is observed that the BER performance of the proposed decoder with No_rand $=50$ iterations enjoys the same performance of $K$-best decoder with $K^{4}=10^{4}$ iterations. Clearly, the performance of the proposed decoder outperforms $K$-best decoder at the same decoding complexity where $K$-best detection algorithm suffers from two main problems which are the expansion and the sorting operations. $K$-best algorithm expands each $K$ retained paths to its $K$ possible children at each level. The previous step requires sorting the children in each layer before selecting the best $K$ paths. Therefore, its decoding complexity increases exponentially with the increase of the value $\mathrm{K}$ where a high decoding complexity is required to enumerate the children nodes especially in the case of large number of transmit antennas and high constellation sizes as shown in Table I, while the complexity of the proposed decoder increases linearly with the value of No_rand. It can be observed from Figures 3, 4, 5 and Table 2 that the proposed decoder using only 200 iterations achieves almost the same performance as the optimal ML decoder which requires $64^{4}=16777216$ iterations. From Table 1 , it is observed for low constellation sizes, that the complexity of the proposed decoder is similar to that of the costly ML decoder. This is also the case if $N_{o}$ rand $=M^{n T x}$. However, the value exponential growth of $M^{n T x}$ with the increase of the number of transmit antennas and the constellation size is generally much larger than the corresponding growth rate of No_rand required to achieve similar performance. Particularly for a large constellation size and a large number of transmit antennas as, e.g., in the case 64-QAM constellations and $n T x=4$ transmit antennas as shown in Table 2, the performance of the proposed decoder using only No_rand $=200$ iterations enjoys similar performance as the optimal ML decoder.

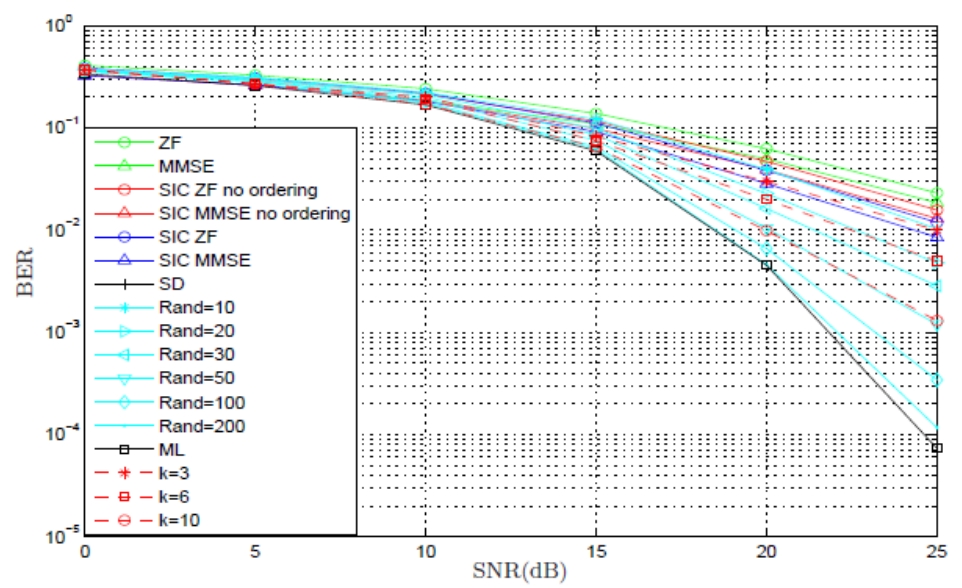

Figure 3. Performance comparison of different decoding schemes using $4 \times 4$ system and 16-QAM constellation 


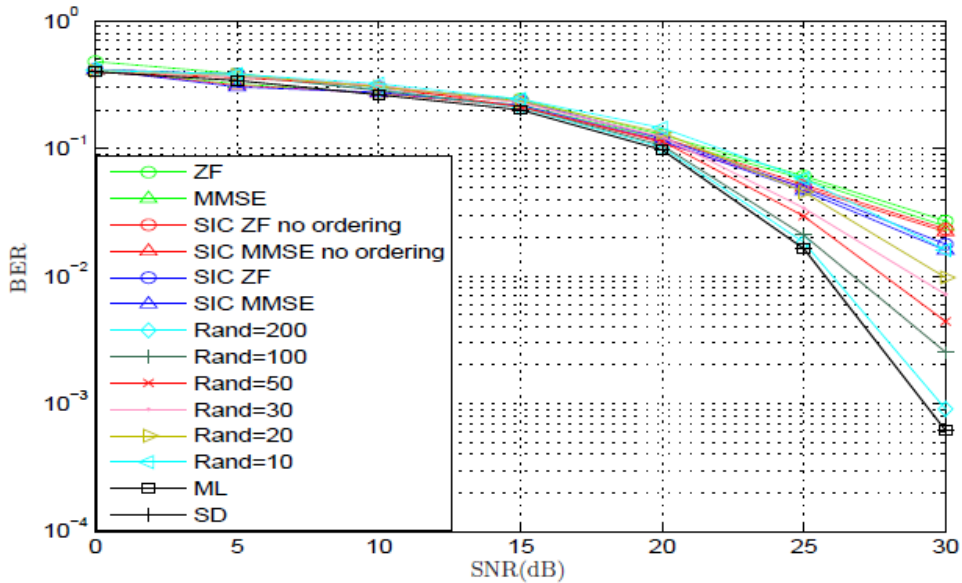

Figure 4. Performance comparison of different decoding schemes using $4 \times 4$ system and 64-QAM constellation

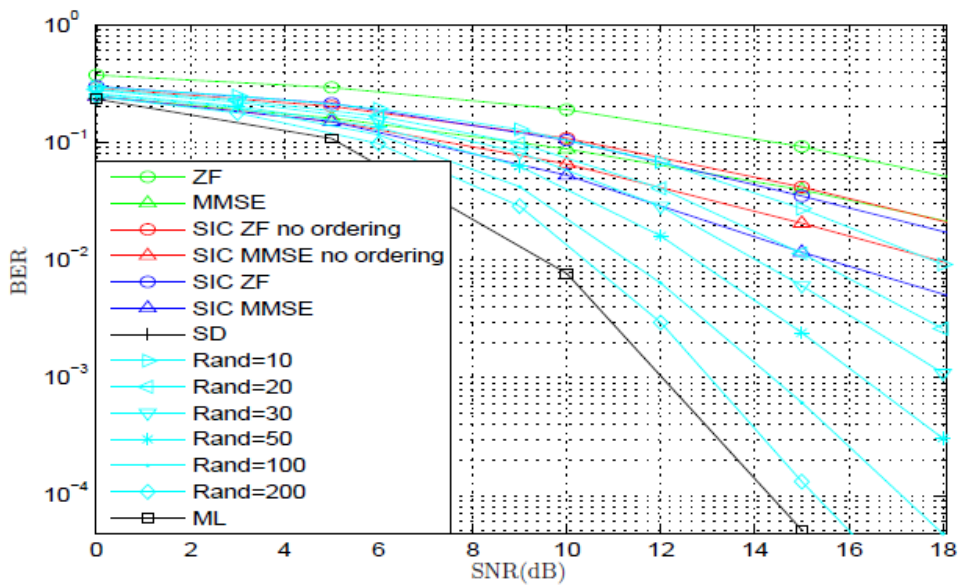

Figure 5. Performance comparison of different decoding schemes using $8 \times 8$ system and 4-QAM constellation

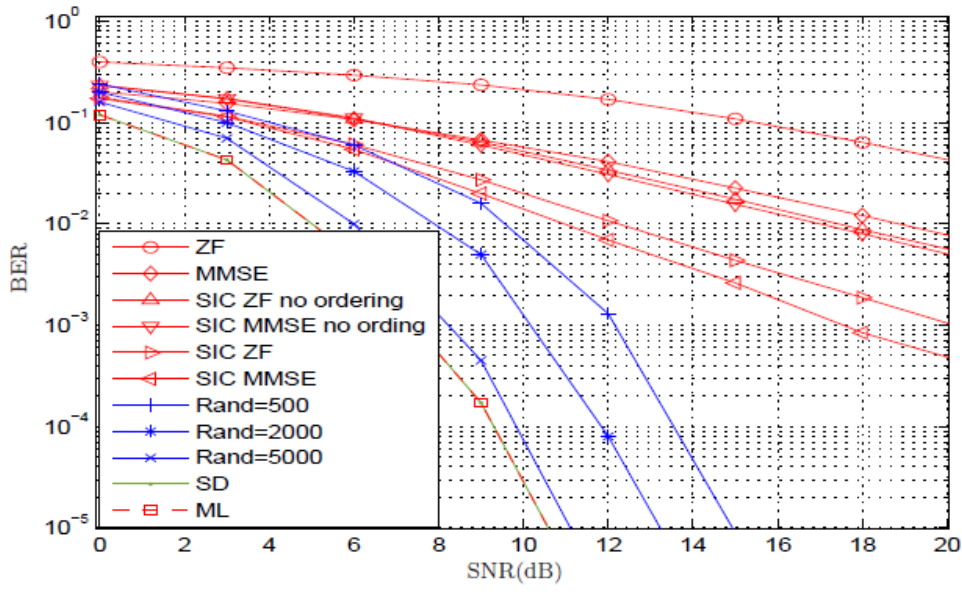

Figure 6. Performance comparison of different decoding schemes using 4-QAM constellation and $20 \times 20$ system 
Table 1. Coding complexity of the investigated MIMO detectors

\begin{tabular}{|c|c|c|c|c|c|}
\hline & $\begin{array}{c}\text { ZF or } \\
\text { MMSE decoder }\end{array}$ & SIC decoder & ML decoder & K-best decoder & The proposed decoder \\
\hline Decoding & $\mathbf{O}\left(\begin{array}{c}(n T x)^{3} \\
+\end{array}\right)$ & $\mathbf{O}\left(\begin{array}{c}(n T x)^{3} \\
+\end{array}\right)$ & $\mathbf{O}\left((n T x)_{+}^{2.38}\right) \times M^{(n T x)}$ & $\mathbf{O}\left((n T x)_{+}^{2.38}\right) \times K^{n T x}$ & $\mathbf{O}\left((n T x)_{+}^{2.38}\right) \times N o \_$rand \\
\hline complexity & $\mathbf{O}\left((n T x)^{2}\right)$ & $\sum_{i=0}^{n T x-1} \mathbf{O}\left((n T x-i)^{2}\right)$ & $\mathbf{O}\left((n T x)^{3}\right)$ & $\begin{array}{c}\sum_{i=0}^{n T x-1} \mathbf{O}\left((n T x)^{2}\right) \\
+ \\
\mathbf{O}\left((n T x)^{3}\right)\end{array}$ & $2 \mathbf{O}\left((n T x)^{3}\right)$ \\
\hline
\end{tabular}

Table 2. Comparison of the relative decoding complexity of the proposed decoder with respect to the ML decoder

\begin{tabular}{ccc}
\hline Scenario & $\begin{array}{c}\text { Number of iterations } \\
\text { No_rand }\end{array}$ & $\begin{array}{c}\text { Relative complexity of } \\
\text { the proposed decoder } \\
\text { w.r.t. the ML decoder }\end{array}$ \\
\hline$(4 \times 4)$ MIMO with 16-QAM & 200 & $0.3052 \%$ \\
$(4 \times 4)$ MIMO with 64-QAM & 200 & $0.0012 \%$ \\
$(8 \times 8)$ MIMO with 4-QAM & 200 & $0.3052 \%$ \\
$(20 \times 20)$ MIMO with 4-QAM & 5000 & $4.5 \times 10^{-7} \%$ \\
\hline
\end{tabular}

\section{EFFECT OF NOISE VARIANCE}

In this section, let us compare the robustness of the proposed decoder with respect to a mismatch between the true and the noise variance at the receiver. In the simulations, let us consider a MIMO system with four transmit and four receive antennas and a true noise variance of $0-\mathrm{dB}$. We assume that the SNR at the receiver amounts to $S N R=P_{t} / \sigma^{2}=12 d B$ in Figure 7 and $S N R=P_{t} / \sigma^{2}=17 d B$ in Figure 8 , and the estimated (presumed) SNR at the receiver side is varied between $0-\mathrm{dB}$ to $20-\mathrm{dB}$. From Figures 7 and 8 , it is observed that for a number of randomization instances exceeding $N \_$rand $=20$ the performance of the proposed algorithm in terms of BER remains approximately constant as the estimated receive SNR is varied across the entire range considered in the simulations. This shows that the proposed algorithm is fairly robust with respect to a mismatch in the noise variance or SNR estimation. This is due to the idea of the proposed decoder which depends on generating random vectors. These random vectors could be far away from the optimal one, however, there is a high probability that some of them will lie very close to it even if the variance of the noise is changed.

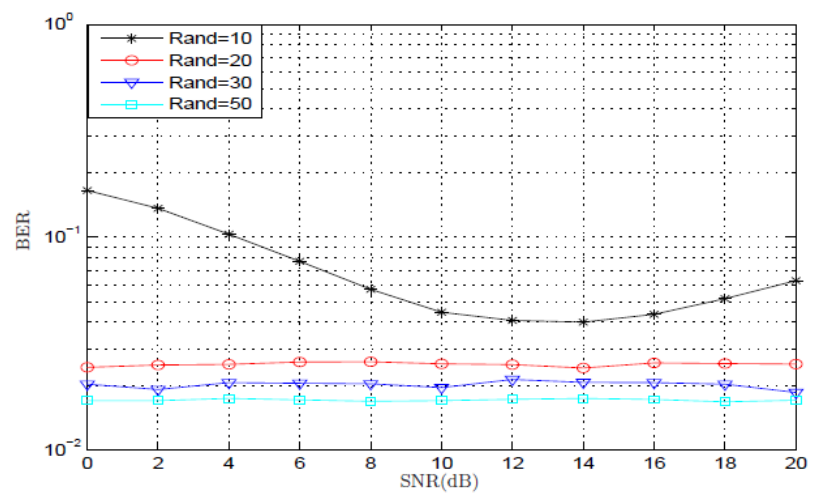

Figure 7. Robustness of the proposed decoder to a mismatch between the true SNR value $\left(P_{t} / \sigma^{2}=12 d B\right)$ and the presumed SNR value in a $4 \times 4$ system using 4-QAM 


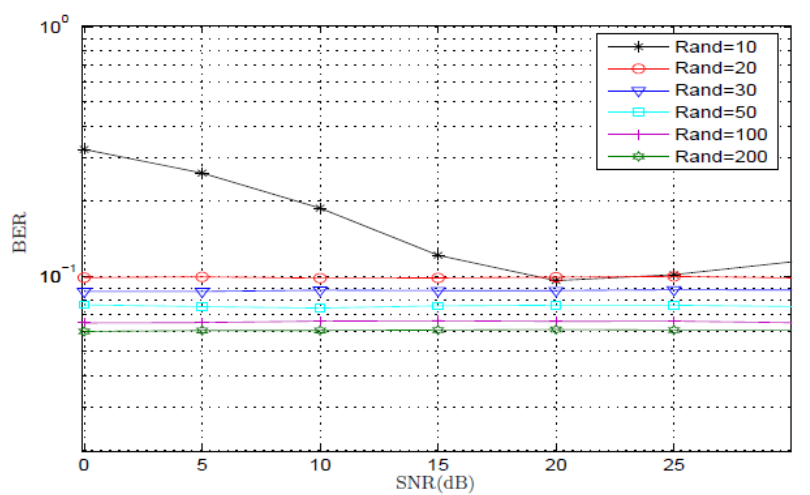

Figure 8. Robustness of the proposed decoder to a mismatch between the true SNR value $\left(P_{t} / \sigma^{2}=17 d B\right)$ and the presumed SNR value in a $4 \times 4$ system using 16-QAM

\section{CONCLUSION}

The proposed decoder appears to find the optimal compromise between competing interests encountered in the implementation of advanced MIMO detectors in practical hardware systems. The proposed detector exhibits a number of desirable properties such as: i) deterministic latency where the proposed decoder exhibits configurable and fully deterministic decoding complexity, which offers the benefit of a fixed decoding complexity, ii) full parameterizable performance/complexity tradeoff where the modification of the number of randomization instances used in the proposed decoder allows to balance at runtime the tradeoff between performance and computational complexity, iii) simple implementation where the proposed algorithm enjoys simple implementation with a minimum requirement of control structures and the proposed detector allows a high degree of parallelization, iv) extension to soft-bit output where the proposed decoder can naturally be extended to create soft-bit outputs as required in modern cellular communication standards.

\section{REFERENCES}

[1] S. Alabed, J. Paredes, and A. B. Gershman, "A simple distributed space-time coded strategy for two-way relay channels," IEEE Transactions on Wireless Communications, pp. 1260-1265, vol. 11, no. 4, April, 2012.

[2] Alex B. Gershman and N. Sidiropoulos, "Space-time processing for MIMO communications," John Wiley and Sons, Ltd, 2005.

[3] S. Alabed, "Performance analysis of differential beamforming in decentralized networks," International Journal of Electrical and Computer Engineering, pp. 1692-1700, vol. 8, no. 3, June 2018.

[4] S. Alabed, "Computationally efficient multi-antenna techniques for multi-user two-way wireless relay networks," International Journal of Electrical and Computer Engineering, pp. 1684-1691, vol. 8, no. 3, June 2018.

[5] S. Alabed, "Performance analysis of two-way DF relay selection techniques," Special Issue on ICT Convergence in the Internet of Things (IoT), Elsevier, pp. 91-95, vol. 2, no. 3, 2016. DOI: 10.1016/j.icte.2016.08.008.

[6] S. Alabed, M. Pesavento, and A. Gershman, "Distributed differential space-time coding techniques for two-way wireless relay networks," In Proceedings of the Fourth IEEE International Workshop on Computational Advances in Multi-Sensor Adaptive Processing (CAMSAP 11), pp. 221-224, San Juan, Puerto Rico, 2011.

[7] S. Alabed, M. Pesavento, and A. Klein, "Distributed differential space-time coding for two-way relay networks using analog network coding," In Proceedings of the 1st European Signal Processing Conference (EUSIPCO'13), Marrakech, Morocco, Sep. 9-13, 2013.

[8] S. Alabed, M. Pesavento, and A. Klein, "Relay selection based space-time coding for two-way wireless relay networks using digital network coding", The Tenth International Symposium on Wireless Communication Systems, TU Ilmenau, Ilmenau, Germany, Aug. 27-30, 2013.

[9] S. Alabed and M. Pesavento, "A simple distributed differential transmit beamforming technique for two- 
way wireless relay networks," In the 16th International IEEE/ITG Workshop on Smart Antennas (WSA 2012), pp. 243-247, Dresden, Germany,2012

[10] A. Schad, S. Alabed, H. Degenhardt, and M. Pesavento, "Bi-directional differential beamforming for multi-antenna relaying," 40th IEEE International Conference on Acoustics, Speech and Signal Processing, 2015.

[11] S. Alabed, M. Pesavento, and A. Klein, "Non-coherent distributed space-time coding techniques for twoway wireless relay networks," EURASIP Special Issue on Sensor Array Processing, 2013.

[12] X. Wen, K. Law, S. Alabed, and M. Pesavento, "Rank-two beamforming for single-group multicasting networks using OSTBC," Proc. of the 7th IEEE Sensor Array and Multichannel Signal Processing Workshop (SAM), pp. 65-68, Jun. 2012.

[13] D. Taleb, S. Alabed, and M. Pesavento, "Optimal general-rank transmit beamforming technique for multicasting service in modern wireless networks using STTC," Proceedings of the 19th International IEEE/ITG Workshop on Smart Antennas (WSA 2015), Ilmenau, Germany, March 2015.

[14] N. Miyazaki, S. Yoshizawa, and Y. Miyanaga, "Low-power dynamic MIMO detection for a 44 MIMOOFDM receiver," IEICE Transactions on Fundamentals of Electronics, Communications and Computer Sciences, vol. E97.A, no. 1, pp. 306-312, 2014.

[15] S. Alabed, J. Paredes, and A. Gershman, "A low complexity decoder for quasi-orthogonal space-time block codes," IEEE Transactions on Wireless Communications, vol. 10, no. 3, March 2011.

[16] M. Neinavaie and M. Derakhtian, "ML performance achieving algorithm with the zero-forcing complexity at high SNR regime," IEEE Tran. on Wireless Comm., vol. 15, no. 7, pp. 4651-4659, July 2016.

[17] M. Ammari and P. Fortier, "Low complexity ZF and MMSE detectors for the uplink MU-MIMO systems with a time-varying number of active users," IEEE Tran. on Vehicular Tech., vol. 66, no. 7, pp. 6586-6590, July 2017 .

[18] S. Ahmed and S. Kim, "Efficient SIC-MMSE MIMO detection with three iterative loops," International Journal of Electronics and Communications, vol. 72, pp. 65-71, Feb. 2017.

[19] X. Zhang, M. Zhang, Q. Zhao, et al., "Comparison of V-BLAST/OSIC algorithm and the QR decomposition algorithm," Int. Conf. on Measurement Information and Control, pp. 1158-1162, October 2013.

[20] K. Wong, C. Tsui, R. Cheng, and W. Mow, "A VLSI architecture of a K-best lattice decoding algorithm for MIMO channels," in Proc. IEEE Int. Symp. Circuits Syst. vol. 32, pp. 273-276, May 2002.

\section{BIOGRAPHY OF AUTHORS}

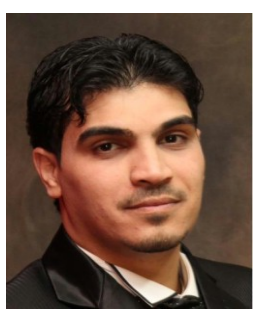

Samer Alabed Samer Alabed joined American University of the Middle East as an assistant professor of electrical engineering in 2015. He received his $\mathrm{PhD}$ degree in electrical engineering and information technology with great honor ("magna cum laude"), from Darmstadt University of Technology, Darmstadt, Germany and his Bachelor and Master degree with great honor. During the last 16 years, he has served as an assistant professor, (post-doctoral) researcher, teaching assistant, and lecturer in several universities in Germany and Middle East and supervised tens of master theses and several PhD students. Dr. Alabed received several awards from IEE, IEEE, DAAD ... etc., where the last one was the best paper award from the International IEEE WSA in March, 2015. Dr. Alabed has worked as a researcher in several universities and companies and was invited to many conferences and workshops in Europe, US, and North Africa. The main idea of his research is to develop advanced DSP algorithms in the area of wireless communication systems and networks including (Massive) MIMO systems, distributed systems, co-operative communications, relay networks, space-time block and trellis coding, differential and blind multi-antenna techniques, MIMO channel estimation, MIMO decoders, channel coding and modulation techniques, distributed communication systems, two-way relaying, baseband communications, multi-carrier transmission (OFDM), modeling of wireless channel characteristics, adaptive beamforming, sensor array processing, transceiver design, multi-user and multi-carrier wireless communication systems, convex optimization algorithms for signal processing communications, channel equalization, and other kinds of distortion and interference mitigation. Further information on his homepage: http://drsameralabed.wixsite.com/samer 